\title{
Des professionnels avec des compétences de leadership
}

\author{
Marie-Paule Fauchère ${ }^{a}$, Bruno Gutknecht ${ }^{b}$, Thomas Heuberger ${ }^{c}$ \\ a Présidente de I'Association romande des assistantes médicales (ARAM) \\ ${ }^{b}$ Avocat, directeur de l'OrTra formation professionnelle des assistantes médicales et secrétaire central de l'Association suisse des assistantes médicales \\ c Dr med., président de l'OrTra formation professionnelle des assistantes médicales
}

L'Association romande des assistantes médicales (ARAM), Médecins de famille Suisse (MFE), l'Association des écoles des professions médicales (SVMB), l'Association suisse des assistantes médicales (SVA), ainsi que la Fédération des médecins suisses (FMH) ont élaboré ensemble une formation complémentaire pour assistante médicale sous forme d'un examen professionnel avec brevet fédéral. Après le retrait du recours déposé par la Fédération suisse des associations d'assistantes médicales (FSAAM) auprès du Secrétariat d'Etat à la formation, à la recherche et à l'innovation (SEFRI), le règlement de l'examen est entré en vigueur le 5 février 2015. La première session d'examen aura lieu en décembre 2015. Pour en savoir plus, veuillez consulter le site www.odamed.ch.

\section{Profils professionnels}

Deux orientations différentes sont prévues pour la profession de coordinateur en médecine ambulatoire, par conséquent l'examen se passe dans les deux orientations.

\section{Coordinatrices en médecine ambulatoire, orientation clinique}

Les coordinatrices en médecine ambulatoire, orientation clinique, travaillent dans un cabinet médical et, sous la supervision du médecin, elles accompagnent des personnes souffrant d'une ou plusieurs maladies chroniques. Tout en tenant compte de la situation actuelle du patient et sur la base de consignes factuelles, elles planifient la prise en charge à venir, stimulent les compétences d'autonomie et de soin des patients et favorisent les soins prodigués par des proches. Elles s'occupent de la coordination des différents services mis à disposition par la commune et contribuent à en simplifier l'accès. Elles connaissent les rôles les plus importants des autres professionnels de la santé et l'étendue de leurs compétences et respectent les limites de leurs propres compétences professionnelles. Dans les rela- tions avec les assurances-maladie et les autres assurances, elles apportent leur contribution en tant que responsables de groupe dans le cadre du Case Management.

\section{Coordinatrices en médecine ambulatoire, orientation gestion}

Les coordinatrices en médecine ambulatoire, orientation gestion, conjuguent l'expérience professionnelle du domaine de la clinique médicale avec le savoir-faire en management. Au sein du cabinet médical, elles occupent une fonction de cadre et sont chargées de la gestion des ressources humaines, des revenus, de ce qui a trait au marketing du cabinet médical et de l'assurance qualité. Dans les relations avec les assurancesmaladie et les autres assurances, elles apportent leur contribution en tant que responsables de groupe dans le cadre du Case Management.

\section{Besoin}

Avec l'obtention d'un brevet fédéral, l'assistante médicale dispose pour la première fois d'une certification de compétences, qualification reconnue au niveau fédéral, qui lui permet de quitter la position de subalterne imposée par le système de formation professionnelle actuel (niveau secondaire II). Cette formation complémentaire au niveau tertiaire lui offre de nouvelles opportunités professionnelles. Permettant d'élargir l'éventail des compétences dans le domaine du Chronic Care Management et le suivi des patients chroniques, là où les compétences du médecin ne sont pas forcément indispensables, la nouvelle fonction de coordinatrices/coordinateurs en médecine ambulatoire, orientation clinique, revêt un grand intérêt pour les médecins de famille. Les négociations avec les autorités sanitaires et les assurances dans le but de pouvoir facturer les prestations des CMA sont en cours.

Les initiateurs du projet de l'Ortramed ont relevé un besoin croissant de professionnels avec des compé- 


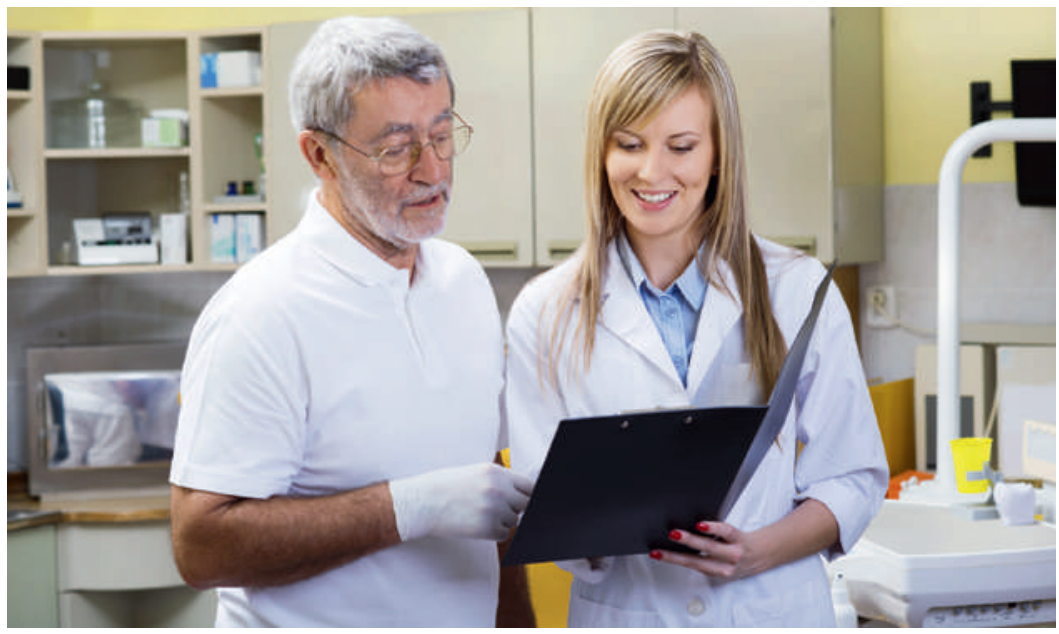

La formation complémentaire au niveau tertiaire offre de nouvelles opportunités professionnelles.
- les personnes qui disposent d'une expérience professionnelle d'au moins 3 ans après la fin de la formation initiale et qui ont pratiqué dans tous les domaines de compétences selon l'ordonnance de la formation professionnelle.

Pour l'examen professionnel, orientation gestion, sont admis

- les assistants médicaux CFC, les assistants médicaux qualifiés, les assistantes médicales DFMS avec autorisation de radiographier, les personnes titulaires d'un certificat de fin d'étude correspondant à un niveau de formation professionnelle équivalente et/ou supérieure;

- les personnes qui disposent d'une expérience professionnelle d'au moins 3 ans après la fin de la formation initiale dans tous les domaines d'un cabinet médical ou d'une institution équivalente.

tences de leadership pour les cabinets de groupe ou les centres médicaux. Il s'agit donc de former des assistantes médicales ayant des qualités de leadership et des compétences de management. Les médecins pourront ainsi déléguer les tâches organisationnelles et administratives afin de se consacrer davantage à leur rôle de diagnosticien.

\section{Formation modulaire}

La formation complémentaire visant à atteindre le niveau du brevet fédéral est construite sur la base d'un enseignement par module d'environ 40 à 60 leçons. Elle contient, pour l'orientation clinique, un approfondissement des connaissances médicales, basé sur la formation initiale des assistantes médicales et, pour l'orientation gestion, des modules du domaine de la gestion d'entreprise et du personnel. L'examen porte sur tous les modules. Les candidats sont évalués sur la présentation d'une étude de cas, lors d'un entretien avec les experts et d'une partie écrite. La formation modulaire coûte environ 10000 francs, frais d'inscription à l'examen professionnel inclus, sans tenir compte d'éventuelles subventions cantonales.

\section{Conditions d'admission}

Pour l'examen professionnel, orientation clinique, sont admis

- les assistants médicaux CFC, les assistants médicaux qualifiés, les assistantes médicales DFMS avec autorisation de radiographier, les personnes titulaires d'un certificat de fin d'étude correspondant à un niveau de formation professionnelle équivalente et/ou supérieure et justifiant de l'autorisation de radiographier;
Pour être admis à l'examen professionnel, les conditions suivantes sont également exigées pour les deux orientations:

- un certificat de maître d'apprentissage;

- un certificat des modules acquis ou certificat d'équivalence.

\section{Modules}

Pour être admis à l'examen du brevet fédéral, l'acquisition d'un nombre défini de modules parmi ceux présentés ci-dessous est requise:

\section{Orientation clinique}

\section{Modules obligatoires}

- Chronic Care Management (module de base I);

- Chronic Care Management (module de base II);

- Management de la qualité au cabinet médical;

- Patient chronique - Diabète.

\section{Deux modules au choix}

- Patient chronique-Maladies rhumatismales;

- Patient chronique - Maladies des voies respiratoires;

- Patient chronique - Maladies cardio-vasculaires;

- Patient chronique-Maladies dégénératives du SNC;

- Traitement de plaies;

- Radiologie élargie.

\section{Orientation gestion}

\section{Modules obligatoires}

- Chronic Care Management (module de base I);

- Chronic Care Management (module de base II); 
- Management de la qualité au cabinet médical;

- Management du cabinet médical;

- Gestion du personnel.

Un module au choix

- Comptabilité;

- Approfondissement en informatique;

- Retraitement de dispositifs médicaux (stérilisation selon Odim);

- Radiologie élargie.

Actuellement pour la Suisse romande, seuls les modules "retraitement de dispositifs médicaux» (reconnu par la SSSH) et «radiologie élargie» (reconnu par l'OFSP, département de radiologie) sont organisés et peuvent faire partie intégrante du brevet fédéral. Les autres modules sont en cours de préparation. Les premiers modules débuteront dans le courant de l'automne 2015 . Les institutions qui proposent des modules, les centres de formation et les fédérations doivent être certifiés par l'OrTra et faire certifier leurs modules.

\section{Cours de remise à niveau}

La validité des modules est limitée à 5 ans. Certains prestataires vont organiser un cours de remise à niveau pour que la date de validité puisse être renouvelée pour l'admission à l'examen professionnel. Pour définir la nécessité d'une remise à niveau de l'un ou l'autre des modules, l'OrTra évaluera si, après 5 ans, le programme de l'enseignement du module ne correspond plus à l'évolution de la pratique au cabinet médical (ex. recherches et nouvelles connaissances dans le domaine du diabète).

\section{Reconnaissance de formation antérieure}

En Suisse romande, le paysage éducatif propose nombre de formations certifiantes, entre autres dans le domaine administratif. Une personne ayant suivi une formation équivalente à l'un des modules du brevet fédéral peut soumettre une demande de reconnaissance d'équivalence à la commission d'assurance qualité de l'Ortramed. Chaque cas sera examiné individuellement moyennant une méthode d'équivalence. La commission déterminera s'il y a lieu ou non d'entrer en matière pour une déduction de module dans le cursus d'étude.
Correspondance: Dr Thomas Heuberger OdAmed

Monbijoustrasse 35

Case postale 6432

CH-3001 Berne

Tél. 0313805458

info[at]odamed.ch
Experts pour les examens professionnels de coordinateurs/coordinatrices en médecine ambulatoire (orientation clinique et orientation gestion)

Les premiers examens professionnels de coordinateurs/coordinatrices en médecine ambulatoire avec brevet fédéral se dérouleront le 3 décembre 2015. Nous recherchons des expertes et experts aux examens pour ce nouveau cursus de formation des assistantes médicales. Toutes les informations concernant les modules et les modalités de l'examen peuvent être consultées sur www.odamed.ch.

La tâche des experts consiste à évaluer les études de cas écrites, à faire passer les entretiens spécialisés et à noter les examens écrits. Le profil de compétences requis est le suivant:

- Formation de médecin avec plusieurs années d'expérience professionnelle en médecine ambulatoire de premier recours, en gestion des soins chroniques et en gestion de la qualité au cabinet;

- Aptitudes pédagogiques et didactiques ou expérience en matière de formation professionnelle;
- Intérêt et engagement pour le développement de la profession d'assistante médicale et du cursus de formation.

Pour se préparer à leur activité, tous les experts participent à une journée de formation de I'Institut fédéral des hautes études en formation professionnelle (IFFP), niveau formation professionnelle supérieure.

Nous recherchons également des mentors qui accompagnent les candidats qui le souhaitent lors de la rédaction de leur étude de cas.

Ces deux activités d'expert et de mentor sont rémunérées par les organisations chargées des examens selon les directives du Secrétariat d'Etat à la formation, à la recherche et à l'innovation (SEFRI).

Pour de plus amples renseignements: Secrétariat de l'OrTra formation professionnelle des assistantes médicales ortramed, Monbijoustr. 35, case postale 6432, 3001 Berne, tél. 031 38054 58, www.odamed.ch; info[at]odamed.ch 